\title{
Cervical and Vulvar
}

\section{Intraepithelial Neoplasia after Treatment with Oral Isotretinoin for Severe Acne Vulgaris}

\author{
M.N. Al Hallak ${ }^{a}$ N. Zouain ${ }^{a, b}$ \\ aUniversity of North Dakota School of Medicine and Health Sciences, \\ ${ }^{b}$ MeritCare Hospital, Roger Maris Cancer Center, Fargo, N. Dak., USA
}

\section{Key Words}

Cervical intraepithelial neoplasia - Vulvar intraepithelial neoplasia - Isotretinoin · Acne vulgaris · Chemoprevention

\begin{abstract}
Oral isotretinoin is the drug of choice for severe acne vulgaris, but its use is still controversial in preventing, treating or stopping the progression of the cervical intraepithelial neoplasia [6-8]. It induces cell differentiation, inhibits cell proliferation, stimulates host immune reaction, inhibits the oncogene expression, augments cellmediated cytotoxicity, and induces apoptosis [5]. The isotretinoin has many side effects including teratogenicity. There is no previous report of developing cervical intraepithelial neoplasia (CIN) or vulvar intraepithelial neoplasia (VIN) after introducing oral isotretinoin to a patient. We are reporting a 37-year-old female with no risk factors for cervical cancer who had developed CIN-I and VIN-I during a 6-month treatment period of oral isotretinoin for her severe acne vulgaris. Interestingly, the patient had complete spontaneous pathologic-proven remission after stopping the isotretinoin. Further case reports are warranted to support this incidence.
\end{abstract}

\section{Introduction}

Oral isotretinoin is a 13-cis retrinoic acid that is useful in the treatment of severe acne vulgaris. It acts primarily by reducing sebaceous gland size and sebum production, and as a result alters skin surface lipid composition [1]. The drug is also antiinflammatory and inhibits comedogenesis [1]. The indications for therapy with oral isotretinoin include severe nodulocystic acne, relapsing acne, scarring acne and acne that improves less than $50 \%$ after 6 months of oral antibiotics treatment $[2,3]$. However, in human studies, the 
role of vitamin A in cancer prevention is still controversial. Meyskens et al. reported complete remission of grade II cervical intraepithelial neoplasia following topical application of retinoic acid in 301 patients [6]. However, Kim et al. have implied in their study that orally administered high doses of 13-cis retrinoic acid seem ineffective in preventing carcinogenesis of the cervical tissue [7]. In addition, Robinson et al. have proven that isotretinoin was not associated with longer time progression of low-grade squamous intraepithelial lesions in HIV-positive patients [8]. Isotretinoin is associated with a number of adverse effects including teratogenicity, cheilitis, dry skin, desquamation, photosensitivity and pruritus. Other side effects including arthralgias, myalgias, hyperostosis, pseudotumor cerebri, decreased night vision, corneal opacities, hepatotoxicity and bone marrow suppression occur infrequently. Hypertriglyceridemia occurs in up to $45 \%$ of patients on isotretinoin therapy [4]. There is no report in the literature of any patient developing cervical intraepithelial neoplasia (CIN) or vulvar intraepithelial neoplasia (VIN) after starting oral isotretinoin. We report a case of a healthy young woman with no risk factors for cervical cancer who developed a CIN-I and a VIN-I 5 months after starting treatment with oral isotretinoin for severe acne vulgaris. Interestingly, the cervical lesion vanished 6 months after stopping isotretinoin therapy. There has been a report that vitamin A induces cell differentiation, inhibits cell proliferation, stimulates host immune reaction, inhibits oncogene expression and angiogenesis, augments cell-mediated cytotoxicity, and induces apoptosis [5]. We are not sure if there is an opposite mechanism in which the isotretinoin will induce neoplasia instead of inhibiting oncogenes.

\section{Case Report}

A 37-year-old healthy non-smoker Caucasian female with no past medical history was treated with oral isotretinoin at a dose of $1 \mathrm{mg} / \mathrm{kg}$ for severe acne vulgaris. The patient was not taking any other medication. She has no family history of cervical cancer. She is married and has a monogamous relationship with her husband. The patient was up-to-date regarding her Papanicolaou (PAP) smear and the last one was 2 years ago and was normal. She has never had a human papilloma virus (HPV) vaccine. Five months after starting the oral isotretinoin she had her already scheduled screening PAP smear and it showed dysplasia. A colposcopy was done which showed 3 small suspicious lesions on the cervix from which the biopsies came back as cervical intraepithelial neoplasia grade I. The pathology was reviewed at two university hospitals which confirmed the results. A vulvoscopy was done and a suspicious lesion was visualized. A biopsy of the lesion was performed. The pathology confirmed a vulvar intraepithelial neoplasia grade I. A HPV reflex test and an in situ hybridization test were performed and both were negative. A complete blood work was done and neither a neutropenia nor an agranulocytosis was diagnosed. Given the fact that the patient has no risk factors to develop cervical carcinoma we started thinking whether the systemic isotretinoin had something to do with the immune system modulation that might have led to the development of the CIN and VIN lesions in this patient. Isotretinoin was stopped and a follow-up colposcopy and vulvoscopy were performed 6 months later. Interestingly, the lesions on the cervix and vulva had completely disappeared. Random biopsies from the cervix were obtained which were negative for any dysplasia or malignancy.

\section{Conclusion}

The ability of isotretinoin to prevent, treat or even to stop the progression of cervical intraepithelial neoplasia is still controversial [6-8]. There is no incidence in the literature of CIN or VIN developing after starting a patient on isotretinoin. The patient that we reported here is the first case in the literature of assumed CIN and VIN induced by isotretinoin given the fact that the dysplasia has reversed after stopping the isotretinoin treatment. Further case reports are warranted to support this assumption. 


\section{References}

1 Ward A, Brogden RN, Heel RC, et al: Isotretinoin: a review of its pharmacological properties and therapeutic efficacy in acne and other skin disorders. Drugs 1984;28:6-37.

2 Ortonne JP: Oral isotretinoin treatment policy. Do we all agree? Dermatology 1997;195:34-37.

-3 Cunliffe WJ, van de Kerkhof PC, Caputo R: Roaccutane treatment guidelines: results of an international survey. Dermatology 1997;194:351-357.

4 Zane LT, Leyden WA, Marqueling AL, Manos MM: A populatin-based analysis of laboratory abnormalities during isotretinoin therapy for acne vulgaris. Arch Dermatol 2006;142:1016-1022.

5 Lotan R: Retinoids in cancer chemoprevention. FASEB J 1996;10:1031-1039.

-6 Meyskens FL Jr, Surwit E, Moon TE, Childers JM, Davis JR, Dorr RT, et al: Enhancement or regression of cervical intraepithelial neoplasia II (moderate dysplasia) with tropically applied all-trans-retinoic acid: a randomized trial. J Natl Cancer Inst 1994;86:539-543.

7 Kim SH, Park TK, Kwon JY: 13-cis-retinoic acid for chemoprevention after colpoconization for cervical intraepithelial neoplasia. Comparative Study. Int J Gynaecol Obstet 2003;81:217-218.

8 Robinson WR, Andersen J, Darragh TM, Kendall MA, Clark R, Maiman M: Isotretinoin for low-grade cervical dysplasia in human immunodeficiency virusinfected women. Obstet Gynecol 2002;99:777-784. 